\title{
A characterization of second order differential operators on finite element spaces
}

\author{
Snorre H. Christiansen*
}

July 25,2003

\begin{abstract}
We describe all operators on scalar finite element spaces which appear as the restriction of a second order (linear) differential operator. More precisely we provide a family of isomorphisms between this space of discrete differential operators and products of some exotic finite element spaces. This provides a unified framework for the Regge calculus of numerical relativity and the Nédélec edge elements of computational electromagnetism.
\end{abstract}

\section{Introduction}

Regge calculus [14] (RC) is an old technique for the construction of discrete variants of the Einstein equations of General Relativity. However it seems to have been more popular as a theoretical tool in quantum gravity (see [15] for references) than as a practical tool for simulating cosmological phenomena. Indeed only a paragraph is devoted to $\mathrm{RC}$ in the recent review by Lehner [11]. Notable exceptions to this impression are the development of Sorkin's parallelizable evolution scheme [4], and computations reported by Gentle [9]. This could indicate a change of tide. However it is acknowledged that $\mathrm{RC}$ would benefit from tighter connections with more classical schemes such as the finite difference, finite volume or finite element methods used by numerical analysts. For instance, due to the possible singularity of gravitational fields, local mesh refinement strategies and/or $h p$ finite elements together with aposteriory estimates would be highly desirable for making the $\mathrm{RC}$ viable as a numerical method. At an even more basic level, error estimates are almost completely absent from RC.

In the finite element community the remark made by Bossavit [6], that the mixed finite elements of Raviart-Thomas [13] and Nédélec [12] can be interpreted as differential forms, called Whitney forms [19], has lead to a

${ }^{*}$ CMA c/o Matematisk Institutt, PB 1053 Blindern, N-0316 Oslo, Norway. 
deaper understanding of these finite element techniques. In particular the discrete version of the DeRham complex has proved useful in proving stability estimates for mixed finite element discretizations, so-called Babuska Brezzi Inf-Sup conditions [3][7], and in analysing the discrete eigenvalue problems of electromagnetism. We refer to [1] and the references therein for more on this. Arnold and Winther have also used it for designing new finite element spaces for elasticity [2]. It is therefore widely believed that by uncovering the algebraic-differential structure of discretization spaces, metric properties such as stability and convergence, could be obtained more easily.

Discrete versions of electromagnetism on simplical complexes compatible with RC have been presented in the physics community by Sorkin [17] and Weingarten [18]. The combinatorics of the electromagnetic part of these theories is that of simplicial cohomology which again corresponds to the interpretation of lowest order mixed finite elements as Whitney forms. The DeRham complex (slightly veiled) is highlighted in both presentations, and is the main argument to justify the construction.

In this article we provide a new type of link between Regge calculus and finite element theory obtained via the tensor product interpretation of bilinear forms arising from differential operators. We hope this approach will be of help in transfering knowledge between the finite element community and the numerical relativity community. Roughly speaking we show that (given a choice of projector and neglecting in this introduction zero order terms), linear forms on Regge elements and Nédélec elements are in one-toone correspondence with respectively the symmetric and the antisymmetric part of discrete second order differential operators on piecewise continuous affine finite elements.

\section{Compatibility of piecewise defined forms}

Let $n$ be a positive integer ( $n=3$ is the most useful case).

Let $V$ denote a vector space with $\operatorname{dim} V \geq n$. Let $M$ denote an $n$ dimensional polyhedral compact submanifold of $V$ without boundary. On $M$ consider a simplicial complex $\mathcal{T}$, whose affine realization in $V$ is nondegenerate, and which generates $M$. More explicitely the simplicial complex $\mathcal{T}$ on $M$ is a set of finite subsets of $M$, with maximal cardinality $n+1$, such that:

$$
\forall F \in \mathcal{T} \forall F^{\prime} \subset F \quad F^{\prime} \in \mathcal{T} .
$$

For $p \leq n$ denote by $\mathcal{T}_{p}$ the subset of $\mathcal{T}$ consisting of $p$-dimensional simplexes (i.e. elements of cardinality $p+1$ ). For $F \in \mathcal{T}_{p}$ denote by $[F]$ the convex envelope of $F$ in $V$. Non degeneracy of $\mathcal{T}$ is the statement that $[F]$ indeed has $p$-dimensional tangential space for $F \in \mathcal{T}_{p}$, and moreover:

$$
\forall F, F^{\prime} \in \mathcal{T} \quad[F] \cap\left[F^{\prime}\right]=\left[F \cap F^{\prime}\right] .
$$


To say that $\mathcal{T}$ generates $M$ is to say that $M$ is the union of the convex envelopes of the $n$-dimensional simplexes of $\mathcal{T}$.

For each $F, F^{\prime} \in \mathcal{T}$ with $F \subset F^{\prime}$ we denote by $i_{F F^{\prime}}$ the canonical injection:

$$
i_{F F^{\prime}}:[F] \rightarrow\left[F^{\prime}\right]
$$

Notice that we have thus associated (a special type of) corner manifolds to each simplex of $\mathcal{T}$ and transition functions between some of these spaces such that if $F, F^{\prime}, F^{\prime \prime} \in \mathcal{T}$ with $F \subset F^{\prime} \subset F^{\prime \prime}$ we have:

$$
i_{F F^{\prime \prime}}=i_{F^{\prime} F^{\prime \prime}} \circ i_{F F^{\prime}} \text {. }
$$

Remark 2.1 In fact the only purpose of $V$ is to provide such corner manifolds and transition functions. One can very well develop an abstract theory in which $V$ is dropped and only these derived objects are kept, subject to the above conditions, just as one can study differentiable manifolds without supposing that they are submanifolds of a vector space. By associating curved corner manifolds to the simplexes instead of affine corner manifolds one can treat the case of smooth $M$.

We denote by $\Gamma^{p}(\mathcal{T})$ the space of piecewise smooth $p$-forms $u$ on $M-$ not necessarily alternating ${ }^{1}$ - satisfying the following compatibility condition along the faces of the cell decomposition of $M$ by $\mathcal{T}$ : For each $F \in \mathcal{T}$ and $F^{\prime}, F^{\prime \prime} \in \mathcal{T}_{n}$ with $F \subset\left(F^{\prime} \cap F^{\prime \prime}\right)$ the following pull-backs are equal:

$$
\left(i_{F F^{\prime}}\right)^{\star}\left(\left.u\right|_{\left[F^{\prime}\right]}\right)=\left(i_{F F^{\prime \prime}}\right)^{\star}\left(\left.u\right|_{\left[F^{\prime \prime}\right]}\right) .
$$

Here as in the rest of this article piecewise smooth means that for each $F \in \mathcal{T}_{n},\left.u\right|_{[F]}$ has an smooth extension to the affine space generated by $F$, and the above property will just be referred to as compatibility. The usual operations defined piecewise preserve compatibility ; more precisely:

Lemma 2.2 Pointwise sums makes $\Gamma^{p}(\mathcal{T})$ into a vector space. Pointwise multiplication my elements of $\Gamma^{0}(\mathcal{T})$, makes $\Gamma^{p}(\mathcal{T})$ into a module.

Lemma 2.3 Let $\otimes_{l o c}$ be the pointwise tensor product acting piecewise on piecewise smooth forms. Then $\otimes$ loc preserves compatibility, that is:

$$
\otimes_{\text {loc }}: \Gamma^{p}(\mathcal{T}) \times \Gamma^{p^{\prime}}(\mathcal{T}) \rightarrow \Gamma^{p+p^{\prime}}(\mathcal{T})
$$

We denote by $\Lambda^{p}(\mathcal{T})$ the subspace of $\Gamma^{p}(\mathcal{T})$ consisting of alternating forms.

Lemma 2.4 Let $\mathrm{d}$ be the exterior derivative acting piecewise on piecewise smooth alternating forms. Then $\mathrm{d}$ preserves compatibility, that is:

$$
\mathrm{d}: \Lambda^{p}(\mathcal{T}) \rightarrow \Lambda^{p+1}(\mathcal{T})
$$

All three lemmas follow from the fact that the involved operations commute with pull-backs.

\footnotetext{
${ }^{1}$ So at a point $x, u(x)$ is a $p$-multilinear form "depending smoothly on $x$ ".
} 


\section{$3 \quad$ Finite Element spaces}

Let $\mathcal{S}$ denote the space of continuous piece-wise affine scalar functions on $M$. Thus we have: $\mathcal{S} \subset \Gamma^{0}(\mathcal{T})$. This space has one degree of freedom per vertex (i.e. element of $\mathcal{T}_{0}$ ).

We will also need $\mathcal{N}$ the Nédélec space of edge elements and $\mathcal{R}$ the Regge space of metrics. We first give some intuitive definitions of these spaces and reinterpret them later on:

- Initially $\mathcal{N}$ was characterized in $3 \mathrm{D}$ Euclidean space as the space of vectorfields which were piecewise of the form $x \mapsto A \times x+B$, and with matching tangential components along the faces (i.e. element of $\mathcal{T}_{2}$ ) of the tetrahedra (i.e. element of $\mathcal{T}_{3}$ ) of the mesh. As is well known this space has one degree of freedom per edge (i.e. element of $\mathcal{T}_{1}$ ). This space is useful because one can define the curl of such vectorfields. It has degrees of freedom associated with the faces of $\mathcal{T}$. This is enough to define the Maxwell action.

- On the other hand in Regge calculus if $M$ is a spacelike slice, $\mathcal{R}$ is defined as follows, given reals $e_{i}$ for each edge. On each element of $\mathcal{T}_{3}$ there is a unique constant metric such that the lenghts of the edges of the tetrahedron are given by the $e_{i}$. This space is useful because one can define the scalar curvature of this structure. It has degrees of freedom associated with the codimension 2 elements of $\mathcal{T}$ which are edges in $3 \mathrm{D}$. This is enough to define the Einstein-Hilbert action.

In fact the definitions which will be useful to us are slightly different (here we don't make any restriction on the dimension $n$ ):

- We interpret $\mathcal{N}$ as a subspace of $\Gamma^{1}(\mathcal{T})$. It is the vector space generated by the elements of the form:

$$
u \mathrm{~d} v-v \mathrm{~d} u, \quad \text { for } \quad u, v \in \mathcal{S} .
$$

- We interpret $\mathcal{R}$ as a subspace of $\Gamma^{2}(\mathcal{T})$. It is the vector space generated by elements of the form:

$$
(1 / 2)\left(\mathrm{d} u \otimes_{l o c} \mathrm{~d} v+\mathrm{d} v \otimes_{l o c} \mathrm{~d} u\right), \quad \text { for } \quad u, v \in \mathcal{S} .
$$

The above symmetrized product of forms will be denoted by $\vee$ in what follows, whereas the standard exterior product will be denoted $\wedge$. Of course by Lemma 2.3 these products preserve compatibility.

In the next section we will use the following choices of basis for these spaces:

Let $\left(\lambda_{i}\right)$ be the standard basis of $\mathcal{S}$, indexed by $\mathcal{T}_{0}$. Its members are continuous piecewise affine functions specified by the condition that $\lambda_{i}(j)=$ $\delta_{i j}$. Chose an orientation of each edge. Thus for each edge $E \in \mathcal{T}_{1}$ we have an origin $E_{0}$ and a target $E_{1}$. The vector $E_{1}-E_{0}$ will be denoted $E_{0} E_{1}$.

Proposition 3.1 The family $\lambda_{E_{0}} \mathrm{~d} \lambda_{E_{1}}-\lambda_{E_{1}} \mathrm{~d} \lambda_{E_{0}}$, for $E \in \mathcal{T}_{1}$ is a basis of $\mathcal{N}$. 
-Proof: This is rather well-known but we include a proof for completeness. Linear independence stems form the fact that for $E, E^{\prime} \in \mathcal{T}_{1}$ which are edges of an $F \in \mathcal{T}_{n}$, the function:

$$
x \mapsto\left(\lambda_{E_{0}} \mathrm{~d} \lambda_{E_{1}}-\lambda_{E_{1}} \mathrm{~d} \lambda_{E_{0}}\right)_{x}\left(E_{0}^{\prime} E_{1}^{\prime}\right),
$$

is constant on $[F]$ and non zero if and only if $E=E^{\prime}$.

On the other hand if $i, j \in \mathcal{T}_{0}$ are not the extremities of an edge then:

$$
\lambda_{i} \mathrm{~d} \lambda_{j}-\lambda_{j} \mathrm{~d} \lambda_{i}=0 .
$$

Hence the proposed family generates $\mathcal{N}$ as defined above.

Proposition 3.2 The family $\mathrm{d} \lambda_{E_{0}} \vee \mathrm{d} \lambda_{E_{1}}$, for $E \in \mathcal{T}_{1}$ is a basis of $\mathcal{R}$.

-Proof: For $E, E^{\prime} \in \mathcal{T}_{1}$ which are edges of an $F \in \mathcal{T}_{n}$ we remark that the function:

$$
x \mapsto\left(\mathrm{d} \lambda_{E_{0}} \vee \mathrm{d} \lambda_{E_{1}}\right)_{x}\left(E_{0}^{\prime} E_{1}^{\prime}, E_{0}^{\prime} E_{1}^{\prime}\right)=\left(\mathrm{d} \lambda_{E_{0}}\right)_{x}\left(E_{0}^{\prime} E_{1}^{\prime}\right)\left(\mathrm{d} \lambda_{E_{1}}\right)_{x}\left(E_{0}^{\prime} E_{1}^{\prime}\right) .
$$

is constant on $[F]$ and non-zero if and only if $E=E^{\prime}$. This proves that the family is linearly independent.

To prove that it spans $\mathcal{R}$ as defined above, it suffices make the two following remarks: For any $i, j \in \mathcal{T}_{0}$ if $i \neq j$ then either $\mathrm{d} \lambda_{i} \vee \mathrm{d} \lambda_{j}=0$ or $i$ and $j$ are the extremities of a common edge. And for any $i \in \mathcal{T}_{0}$ we have:

$$
\mathrm{d} \lambda_{i} \vee \mathrm{d} \lambda_{i}=\sum_{j \neq i} \mathrm{~d} \lambda_{i} \vee \mathrm{d}\left(1-\lambda_{j}\right)=-\sum_{j \neq i} \mathrm{~d} \lambda_{i} \vee \mathrm{d} \lambda_{j}
$$

This completes the proof.

We will also use the following notation: for any integer $q$, we denote by $\mathcal{S}^{q}$ the subspace of $\Gamma^{0}(M)$ generated by functions of the form:

$$
u_{1} u_{2} \cdots u_{q}, \quad \text { for } \quad u_{i} \in \mathcal{S} .
$$

This space is nothing but the space of continuous piecewise polynomials of degree at most $q$.

\section{Discrete second order differential operators}

By the invariance of the Sobolev space $\mathrm{H}^{1}$ under Lipshitz isomorphisms one can define $\mathrm{H}^{s}(M)$ for $s \in[-1,1]$.

We then define a second order operator on $M$ to be any continuous operator $A: \mathrm{H}^{1}(M) \rightarrow \mathrm{H}^{-1}(M)$. To any second order operator $A$ we can associate a (unique) continuous bilinear form $a$ on $\mathrm{H}^{1}(M)$ by:

$$
a(u, v)=\langle A u, v\rangle .
$$


We shall say that such bilinear forms are second order. Yet another way to describe such an objet is via tensor products: For any continuous bilinear form $a$ on $\mathrm{H}^{1}(M)$ there is a unique continuous linear form $l$ on $\mathrm{H}^{1}(M) \hat{\otimes} \mathrm{H}^{1}(M)$ such that:

$$
l(u \otimes v)=a(u, v) .
$$

For any second order operator $A$ we say that $A$ is differential if it preserves the support:

$$
\forall u \in \mathrm{H}^{1}(M) \quad \operatorname{supp} A u \subset \operatorname{supp} u \text {. }
$$

We also use this terminology for the associated bilinear form $a$, and the associated linear form $l$. Thus, if $l$ is a continuous linear form on $\mathrm{H}^{1}(M) \hat{\otimes} \mathrm{H}^{1}(M)$, $l$ is differential if and only if $l$ vanishes on the closed linear span $W$ of the elements of the form:

$$
u \otimes v \quad \text { with } \quad u, v \in \mathrm{H}^{1}(M) \quad \text { and } \quad \operatorname{supp} u \cap \operatorname{supp} v=\emptyset .
$$

From the continuous point of view the question we are asking is : what does the polar of $W$ look like ? I don't know, but in the discrete setting we have the following results:

Proposition 4.1 For any bilinear form $b$ on $\mathcal{S}$ the following are equivalent:

- $b$ is the restriction to $\mathcal{S}$ of a second order differential bilinear form.

- there exists a linear form $l_{0}$ on $\mathcal{S}^{2}$, a linear form $l_{1}$ on $\mathcal{N}$ and a linear form $l_{2}$ on $\mathcal{R}$ such that:

$$
\forall u, v \in \mathcal{S} \quad b(u, v)=l_{0}(u v)+l_{1}(u \mathrm{~d} v-v \mathrm{~d} u)+l_{2}(\mathrm{~d} u \vee \mathrm{d} v) .
$$

-Proof : Let $\mathcal{B}$ be the space of bilinear forms $b$ on $\mathcal{S}$ satisfying the first assertion. Suppose now $b \in \mathcal{B}$. Remark first that if $i, j \in \mathcal{T}_{0}$ are not two extremities of a common edge then supp $\lambda_{i} \cap \operatorname{supp} \lambda_{j}$ does not contain an $[F]$ for $F \in \mathcal{T}_{n}$, hence $\lambda_{j}$ is limit in $\mathrm{H}^{1}(M)$ of functions with support disjoint from $\operatorname{supp} \lambda_{i}$, so:

$$
b\left(\lambda_{i}, \lambda_{j}\right)=0 .
$$

Thus the matrix of $b$ in the basis $\left(\lambda_{i}\right)$ of $\mathcal{S}$ can have non-zero entries only on the couples $\left(E_{0}, E_{1}\right)$ and $\left(E_{1}, E_{0}\right)$ for $E \in \mathcal{T}_{1}$, and on the diagonal. Therefore the symmetric subspace $\mathcal{B}_{s}$ of $\mathcal{B}$ has dimension at most $\# \mathcal{T}_{0}+$ $\# \mathcal{T}_{1}=\operatorname{dim} \mathcal{S}^{2}$ whereas the antisymmetric subspace $\mathcal{B}_{a}$ has dimension at most $\# \mathcal{T}_{1}=\operatorname{dim} \mathcal{R}$. On the other hand one the checks that the maps:

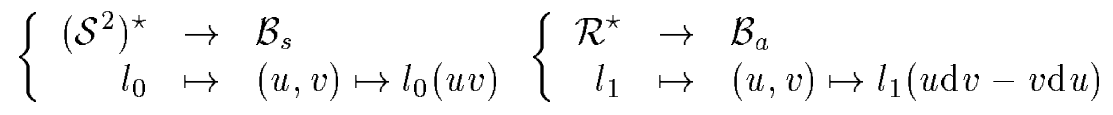


are injective. By the above dimension bounds they are surjective. It follows that the first assertion implies the second.

To show the converse, suppose we have $\left(l_{0}, l_{1}, l_{2}\right)$ as in the second assertion. Then, by the standard theorems, for each $i, l_{i}$ can be extended to a continuous linear form on the completion of $\Gamma^{i}(M)$ with respect to $\mathrm{L}^{1}(M)$ norms. These extensions provide a continous linear form on $\mathrm{H}^{1}(M) \hat{\otimes} \mathrm{H}^{1}(M)$ which is differential.

Such a form will be called a discrete second order differential bilinear form. We denote by $\mathcal{B}$ the vector space they constitute. The above representation of elements of $\mathcal{B}$ by linear forms is not unique as a simple dimension count shows. We also showed in the proof that $l_{2}$ is not needed. But more interestingly we have:

Proposition 4.2 For each projector $P$ in $\mathcal{S}^{2}$ with range $\mathcal{S}$ we have an isomorphism $\Upsilon: \mathcal{S}^{\star} \times \mathcal{N}^{\star} \times \mathcal{R}^{\star} \rightarrow \mathcal{B}$ given by $\Upsilon\left(l_{0}, l_{1}, l_{2}\right)=b$ defined by :

$$
b(u, v)=l_{0}(P(u v))+l_{1}(u \mathrm{~d} v-v \mathrm{~d} u)+l_{2}(\mathrm{~d} u \vee \mathrm{d} v) .
$$

-Proof: The map $\Upsilon$ is linear and as we remarked in the proof of Proposition 4.1 the dimension of the target space is less or equal to that of the origin space. Therefore it suffices to show that $\Upsilon$ is injective.

Suppose $\Upsilon\left(l_{0}, l_{1}, l_{2}\right)=0$. Then both the symmetric and antisymmetric part of $\Upsilon\left(l_{0}, l_{1}, l_{2}\right)$ are 0 . The last condition implies $l_{1}=0$. Therefore we have:

$$
\forall u, v \in \mathcal{S} \quad l_{0}(P(u v))+l_{2}(\mathrm{~d} u \vee \mathrm{d} v)=0 .
$$

For $u=1$ and $v \in \mathcal{S}$ we have $P(u v)=v$ and $\mathrm{d} u=0$, from which we deduce $l_{0}=0$. Therefore we also have $l_{2}=0$.

\section{References}

[1] D.N. ARnold : Differential complexes and numerical stability; Plenary address delivered at ICM 2002, International Congress of Mathematicians, Beijing 2002.

[2] D.N. Arnold, R. Winther : Mixed finite elements for elasticity ; Numer. Math., Vol. 92, No. 3, p. 401-419, 2002.

[3] I. Babuska : Error bounds for the finite element method; Numer. Math., Vol. 16, p. 322-333, 1971.

[4] J.W. Barrett, M. Galassi, W.A. Miller, R.D. Sorkin, P.A. Tuckey, R.M. Williams : Parallelizable implicit evolution scheme for Regge calculus ; Internat. J. Theoret. Phys., Vol. 36, No. 4, p. 815839, 1997. 
[5] D. Boffi : A note on the DeRham complex and a discrete compactness property ; Appl. Math. Lett., Vol. 14, No. 1, p. 33-38, 2001.

[6] A. Bossavit : Mixed finite elements and the complex of Whitney forms ; The mathematics of finite elements and applications, VI (Uxbridge, 1987), p. 137-144, Academic Press, London, 1988.

[7] F. BREzzI : On the existence, uniqueness and approximation of saddlepoint problems arising from Lagrangian multipliers ; RAIRO Anal. Numér., Vol. 8, No. R-2, p. 129-151, 1974.

[8] F. Brezzi, M. Fortin : Mixed and hybrid finite element methods ; Springer-Verlag, 1991.

[9] A. P. Gentle : Regge calculus: a unique tool for numerical relativity; Gen. Relativity Gravitation, Vol. 34, No. 10, p. 1701-1718, 2002.

[10] R. Hiptmair : Canonical construction of finite elements ; Math. Comp., Vol. 68, No. 228, p. 1325-1346, 1999.

[11] L. Lehner : Numerical relativity: a review ; Class. Quantum Grav., Vol. 18, p. 25-86, 2001.

[12] J.-C. NÉDÉLEC : Mixed finite elements in $\mathbb{R}^{3}$; Numer. Math., Vol. 35, p. 315-341, 1980.

[13] P.A. Raviart, J.-M. Thomas : A mixed finite element method for 2nd order elliptic problems; p. 292-315 in I. Galligani, E. Magenes (ed): Mathematical aspects of the finite element method ; Lecture Notes in Math., Vol. 606, Springer-Verlag, Berlin and New York, 1977.

[14] T. RegGe : General relativity without coordinates ; Nuovo Cimento, Vol. 10, No. 19, p. 558-571, 1961.

[15] T. Regge, R.M. Williams : Discrete structures in gravity; J. Math. Phys., Vol. 41, No. 6, p. 3964-3984, 2000.

[16] G. DeRham : Variétés différentiables. Formes, courants, formes harmoniques (Third edition) ; Publications de l'Institut de Mathématique de l'Université de Nancago, III. Actualités Scientifiques et Industrielles, No. 1222b. Hermann, Paris, 1973.

[17] R. Sorkin : The electromagnetic field on a simplicial net; J. Math. Phys., Vol. 16, No. 12, p. 2432-2440, 1975.

[18] D. Weingarten : Geometric formulation of electrodynamics and general relativity in discrete space-time; J. Math. Phys., Vol. 18, No. 1, p. 165-170, 1977. 
[19] H. Whitney : Geometric integration theory ; Princeton University Press, Princeton, N. J., 1957. 